\title{
MINIMAL ISOMETRIC IMMERSIONS OF INHOMOGENEOUS SPHERICAL SPACE FORMS INTO SPHERES- A NECESSARY CONDITION FOR EXISTENCE
}

\author{
CHRISTINE M. ESCHER
}

\begin{abstract}
Although much is known about minimal isometric immersions into spheres of homogeneous spherical space forms, there are no results in the literature about such immersions in the dominant case of inhomogeneous space forms. For a large class of these, we give a necessary condition for the existence of such an immersion of a given degree. This condition depends only upon the degree and the fundamental group of the space form and is given in terms of an explicitly computable function. Evaluating this function shows that neither $L(5,2)$ nor $L(8,3)$ admit a minimal isometric immersion into any sphere if the degree of the immersion is less than 28 , or less than 20 , respectively.
\end{abstract}

\section{INTRODUCTION}

Let $M$ be an $n$-dimensional compact Riemannian manifold and $S^{N}(r)$ a sphere of dimension $N$ and radius $r$. A fundamental result of T. Takahashi [T] is the following theorem:

Theorem (Takahashi). Let $M$ be an $n$-dimensional compact Riemannian manifold and $f: M \rightarrow \mathbb{R}^{N}$ an isometric immersion. Then $f$ is a minimal isometric immersion into a round sphere if and only if all components of $f$ are eigenfunctions of the Laplace operator on $M$ with respect to the same eigenvalue.

Therefore the main idea in constructing minimal isometric immersions of a manifold $M$ into a sphere is to find eigenvalues of the Laplacian on $M$ of sufficiently high multiplicity in order to provide the coordinate functions of the immersions.

Another result of Takahashi [T] is that all isotropy irreducible homogeneous Riemannian manifolds, i.e. manifolds $M=G / H$ whose isotropy group $H$ acts irreducibly on the tangent space, do admit such immersions. To see this, we consider the eigenspace $E_{\lambda}$ to a fixed eigenvalue $\lambda \neq 0$. On $E_{\lambda}$ we have the inner product induced by that of $L^{2}(M)$ and the group $G$ acts on $E_{\lambda}$ by isometries. Let $\left\{\phi_{1}, \ldots, \phi_{N}\right\}$ be an orthonormal basis of $E_{\lambda}$ and let $\phi=\left(\phi_{1}, \ldots, \phi_{N}\right): M \rightarrow \mathbb{R}^{N}$. Then $\sum d \phi_{i}^{2}$ on the one hand can be regarded as the inner product on $E_{\lambda}$ and on the other hand as the metric on $M$ which is the pull back of the standard metric on $\mathbb{R}^{N}$ under $\phi$. In the first interpretation it is clear that $\sum d \phi_{i}^{2}$ must be invariant under the action of $G$ and hence the metric $\sum d \phi_{i}^{2}$ on $M$ must be as well. But then $\sum d \phi_{i}^{2}$ must be a multiple of the given metric on $M$ as both are invariant

Received by the editors August 22, 1995.

1991 Mathematics Subject Classification. Primary 53C42.

Key words and phrases. Minimal isometric immersions, inhomogeneous spherical space forms.

(C)1996 American Mathematical Society 
under the irreducible action of the isotropy group $H$. This multiple cannot be zero as the functions $\phi_{i}$ are not constant. Therefore, after multiplying the metric on $M$ by a constant, $\phi: M \rightarrow \mathbb{R}^{N}$ is an isometric immersion, which by our first stated theorem of Takahashi $[\mathrm{T}]$ gives rise to a minimal isometric immersion into a sphere. This immersion is called the standard minimal isometric immersion of degree $d$ if $\lambda$ is the $d$ th nonzero eigenvalue.

We call two immersions congruent if they differ by an isometry of the ambient space. Note that a different choice of orthonormal basis for $E_{\lambda}$ gives rise to a congruent immersion.

The fundamental example of a homogeneous Riemannian manifold is the $n$ dimensional sphere, realized as the homogeneous space $S O(n+1) / S O(n)$. The eigenfunctions of $S^{n}(1)$ are simply the restrictions of harmonic homogeneous polynomials on $\mathbb{R}^{n+1}$ to $S^{n}(1)$. All the harmonic homogeneous polynomials of degree $g$ restrict to eigenfunctions on $S^{n}$ with the same eigenvalue $\lambda_{g}=g(g+n-1)$ and the dimension of this eigenspace is equal to $N_{g}=(2 g+n-1)(g+n-2) ! /(g !(n-1) !)$. For odd $g$, the standard minimal isometric immersion gives rise to a minimal isometric embedding of $S^{n}$ into $S^{N_{g}-1}\left(\sqrt{n / \lambda_{g}}\right)$. For even $g$, all components of the immersion are invariant under the antipodal map and we get a minimal isometric embedding of $\mathbb{R} P^{n}$ into $S^{N_{g}-1}\left(\sqrt{n / \lambda_{g}}\right)$.

In 1967, E. Calabi $[C]$ showed that every minimal isometric immersion of the 2-sphere of radius $1, S^{2}(1)$, into some $N$-sphere of radius $r, S^{N}(r)$, is congruent to one of the standard eigenspace immersions. In [DW2] the space of all minimal isometric immersions of $S^{n}(1)$ into $S^{N}(r)$ was examined in some detail, and it was shown that for $n>2$ there are many minimal isometric immersions besides the ones described above. If we fix $r=\sqrt{n / \lambda_{g}}$, or equivalently only consider harmonic homogeneous polynomials of degree $g$, then these minimal isometric immersions (up to congruence of the ambient space) are parametrized by a compact convex body $B_{g}$ in a finite dimensional vector space. In [DW2] it is shown that for $n=2$ and any $g$ and for $g=2,3$ and any $n$, the space $B_{g}$ is a point, i.e. any such minimal isometric immersion is congruent to the standard one. A very recent development is the finding of the exact dimension of this convex body by Gabor Toth [To]. For further references on this matter, see [DZ], [DW1], [DW2], [L], [T].

From the description of the convex body $B_{g}$ it follows immediately that its interior points correspond to isometric immersions which use a full basis of $E_{\lambda}$ as their components. As was observed in [WZ], one can show that these immersions are $S O(n+1)$-equivariant immersions into $\mathbb{R}^{N_{g}}$. But the only space forms that contain all of $S O(n+1)$ in their isometry groups are $S^{n}$ and $\mathbb{R} P^{n}$. Hence the image of the immersion must be $S^{n}$ for $g$ odd and $\mathbb{R} P^{n}$ for $g$ even and all other space form images must correspond to boundary points of $B_{g}$.

P. $\mathrm{Li}[\mathrm{L}]$ generalized the above parametrization result of [DW2] to isotropyirreducible homogeneous spaces and also claimed that the image of a minimal isometric immersion of an isotropy-irreducible homogeneous space must be again an isotropy-irreducible homogeneous space. He went on to apply this theorem to the case where $M$ is also a sphere, and concluded that the image of a minimal isometric immersion of a sphere into a sphere must be either a sphere or a real projective space. This would of course imply that there exists no minimal isometric immersion of a lens space or any other more complicated spherical space form into a sphere. That this is indeed not correct was first observed by K. Mashimo [Ma1], who gave 
an example of a minimal isometric immersion of $S^{3}(1)$ into $S^{6}\left(\frac{1}{4}\right)$, whose image is at least a 6 -fold subcover of $S^{3}$ (he did not identify the image completely).

Later, in [WZ], M. Wang and W. Ziller showed that certain quotients of $S^{3}$ (by the so-called binary polyhedral groups) are also isotropy irreducible, and so by the above mentioned theorem of Takahashi, the polyhedral manifolds $S^{3} / T^{*}, S^{3} / O^{*}$ and $S^{3} / I^{*}$ admit minimal isometric immersions into spheres.

This then raises the question of just which spherical space forms do admit minimal isometric immersions or embeddings into spheres.

Spherical space forms, i.e. compact manifolds of constant curvature +1 , have been completely classified [W]. There are two types-homogeneous and inhomogeneous, i.e. those space forms which admit a differentiable transitive left operation of a group $G$ and those which do not. For the homogeneous spherical space forms D. DeTurck and W. Ziller [DZ] showed the following:

Theorem [DZ]. Every homogeneous spherical space form admits a minimal isometric embedding into a standard sphere.

However, the classification shows that very few space forms are actually homogeneous, see [W, Theorem 2.7.1] for a description. Thus a natural question which arises is whether the same theorem is true in the inhomogeneous case.

Denote by $L(p, q)$ the three dimensional lens space generated by the standard action of $\mathbb{Z}_{p}$ on $S^{3}$. Such a lens space is inhomogeneous if and only if $q \not \equiv \pm 1$ mod $p$. The main theorem of this paper gives a necessary condition for the existence of minimal isometric immersions of three dimensional inhomogeneous space forms into spheres. We first show

Theorem 1. The only three dimensional inhomogeneous spherical space forms which cannot be covered by an inhomogeneous lens space are

1. $S^{3} / G$ where $G \cong T$ is a diagonal subgroup of index three in $\phi\left(\tilde{\mathbb{Z}}_{3} \times \tilde{T}\right)$. (Here we use the same notation as in $[\mathrm{S}]$, see also Section 2.)

2. $S^{3} / G$ where $G \cong D_{n}$ is a diagonal subgroup of index two in $\phi\left(\tilde{\mathbb{Z}}_{2} \times \tilde{D}_{n}\right)$.

Let $\mathfrak{U}(p, q)$ be the function of two natural numbers $p$ and $q$ with image in the natural numbers which will be explained in detail in section 4.3.8.

Theorem 2. If $g<\mathfrak{U}(p, q)$, then the three dimensional inhomogeneous lens space $L(p, q)$ does not admit a minimal isometric immersion of degree $g$ into any sphere.

Notation. We will call an inhomogeneous space form a $p q$-space form if it can be covered by some inhomogeneous lens space $L(p, q)$.

As a consequence of the above two theorems we obtain

Corollary. If $g<\mathfrak{U}(p, q)$, then no three dimensional pq-space form admits a minimal isometric immersion of degree $g$ into a sphere.

Observe that the corollary follows directly from Theorems 1 and 2. Indeed, Theorem 1 tells us that except for cases (1) and (2) every inhomogeneous spherical space form $S^{3} / G$ can be covered by an inhomogeneous lens space $L(p, q)$, where $\operatorname{gcd}(p, q)=1$ and $q \not \equiv \pm 1 \bmod p$. Now assume that $g<\mathfrak{U}(p, q)$ and that there exists a minimal isometric immersion of degree $g$ of an inhomogeneous $p q$-space 
form $S^{3} / G$ into a sphere $S^{N}$. Then we have

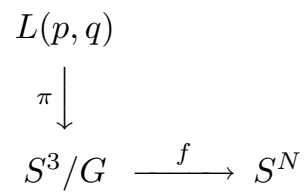

and $f \circ \pi$ is a minimal isometric immersion of degree $g$ with $g<\mathfrak{U}(p, q)$ of $L(p, q)$ into $S^{N}$. But this is not possible by Theorem 2 .

In a sequel to this paper we will analyze the function $\mathfrak{U}(p, q)$. The goal is to show that the function $\mathfrak{U}$ increases with increasing $p$. Hence a calculation for the smallest $p$ will show:

Conjecture 1. If $g<28$ and $p$ is odd, then no pq-space form admits a minimal isometric immersion of degree $g$ into a sphere for any $q$.

If $g<20$ and $p$ is even, then no pq-space form admits a minimal isometric immersion of degree $g$ into a sphere for any $q$.

Example. Computations show that $\mathfrak{U}(5,2)=28$ and $\mathfrak{U}(8,3)=20$, thus there do not exist minimal isometric immersions of $L(5,2)$ into any sphere if $g<28$ and of $L(8,3)$ if $g<20$.

In this paper we will also generalize Theorem 2 to a necessary and sufficient condition for existence:

Conjecture 2. There exists a minimal isometric immersion of degree $g$ of a three dimensional pq-space form into a sphere if and only if $g \geq \mathfrak{U}(p, q)$ and the linear system associated to the isometry condition admits a nonnegative solution.

Remark. Calculations have shown that there do exist solutions of the linear system in degrees 20 and 28 for $L(8,3)$ and $L(5,2)$ respectively, but they are not nonnegative.

The author acknowledges the support of the National Science Foundation and would also like to thank Wolfgang Ziller for pointing out a mistake in an earlier version of this paper. The referee is heartily thanked for a very thorough reading.

\section{Classification of SPherical SPACE FORMS}

In 1930 W. Threlfall and H. Seifert [TS] classified all three dimensional spherical space forms. We will use a more recent discussion of their work by P. Scott [S].

The classification of three dimensional spherical space forms is equivalent to classifying all finite subgroups of $S O(4)$ which act freely on $S^{3}$. The classification includes the well known class of lens spaces. Recall the definition of a lens space. Consider $S^{3}=\left\{\left(z_{1}, z_{2}\right) \in \mathbb{C}^{2} \mid z_{1} \bar{z}_{1}+z_{2} \bar{z}_{2}=1\right\}$. Then, given $q \in \mathbb{Z}, \mathbb{Z}_{p}$ acts on $S^{3}$ as $\left(z_{1}, z_{2}\right) \mapsto\left(e^{\frac{2 \pi \imath}{p}} z_{1}, e^{\frac{2 \pi \imath q}{p}} z_{2}\right)$. The action is free if $\operatorname{gcd}(p, q)=1$ and $L(p, q)=S^{3} / \mathbb{Z}_{p}$. This lens space is inhomogeneous if and only if $q \not \equiv \pm 1 \bmod p$. See $[\mathrm{W}]$ for a proof.

To describe the other space forms, regard $S^{3}$ as the group of unit quaternions:

$S^{3}=\left\{q=u+\left.\jmath v|u, v \in \mathbb{C}| q\right|^{2}=,|u|^{2}+|v|^{2}\right\}$. One can show that the center of $S^{3}$ is $\{+1,-1\}$. An important fact for the classification is an isomorphism between $S O(4)$ and a quotient of $S^{3} \times S^{3}$ by $\mathbb{Z}_{2}$. Recall that $S^{3}$ acts on itself by left and 
right multiplication and that these actions commute and are both isometries. Define a homomorphism

$$
\begin{aligned}
\phi: S^{3} \times S^{3} & \longrightarrow S O(4) \\
\left(q_{1}, q_{2}\right) & \longmapsto\left(x \mapsto q_{1} x q_{2}^{-1}\right),
\end{aligned}
$$

where, of course, $\left(x \mapsto q_{1} x q_{2}^{-1}\right)$ is an isometry of $S^{3}$. The kernel of $\phi$ has order two and its only non-trivial element is $(-1,-1)$. Also, the image of $\phi$ is all of $S O(4)$. Note that the isometry $\alpha(x)=q_{1} x q_{2}^{-1}$ has a fixed point if and only if $q_{1}$ and $q_{2}$ are conjugate. As an example, consider any finite subgroup $G \subset S^{3}$. Then $G \times\{e\}$ or $\{e\} \times G$ act freely on $S^{3}$. These are precisely the homogeneous space forms in dimension $3,[\mathrm{~W}]$.

As a second example, consider $\mathbb{Z}_{r} \times \mathbb{Z}_{s} \subset S^{3} \times S^{3}$, where $\mathbb{Z}_{r}$ is generated by $e^{\frac{2 \pi \imath}{r}}$ and $\mathbb{Z}_{s}$ is generated by $e^{\frac{2 \pi \imath}{s}}$. If there exist $q_{1} \in \mathbb{Z}_{r}$ and $q_{2} \in \mathbb{Z}_{s}$ such that $q_{1}$ and $q_{2}$ are conjugate in $S^{3}$, then $q_{1}=q_{2}$ or $q_{1}=q_{2}^{-1}=\bar{q}_{2}=\jmath q_{2} \jmath^{-1}$ and hence $\mathbb{Z}_{r} \cap \mathbb{Z}_{s} \neq\{e\}$ or equivalently $\operatorname{gcd}(r, s) \neq 1$. Hence if $r$ and $s$ are relatively prime, $\phi\left(\mathbb{Z}_{r} \times \mathbb{Z}_{s}\right) \cong \mathbb{Z}_{r} \times \mathbb{Z}_{s} \cong \mathbb{Z}_{r s}$ acts freely on $S^{3}$ and we claim that this corresponds exactly to a lens space. Indeed

$$
\begin{aligned}
e^{\frac{2 \pi \imath}{r}}(u+\jmath v) e^{-\frac{2 \pi 2}{s}} & =e^{\frac{2 \pi \imath}{r}} u e^{-\frac{2 \pi \imath}{s}}+\jmath e^{-\frac{2 \pi \imath}{r}} v e^{-\frac{2 \pi 2}{s}} \\
& =e^{\frac{2 \pi \imath(s-r)}{r s}} u+\jmath e^{\frac{2 \pi \imath(-r-s)}{r s}} v .
\end{aligned}
$$

If $r=1$ or $s=1$, this clearly corresponds to a homogeneous lens space, e.g. to $L(s, 1)$ if $r=1$. In the case of $r \neq 1$ and $s \neq 1$ the fact that $r$ and $s$ are relatively prime implies that there exist integers $k$ and $l$ with $-k r+l s=1$. We can replace the generators $e^{\frac{2 \pi \imath}{r}}$ and $e^{\frac{2 \pi \imath}{s}}$ by $e^{\frac{2 \pi \imath l}{r}}$ and $e^{\frac{2 \pi \imath k}{s}}$ as $\operatorname{gcd}(l, r)=1$ and $\operatorname{gcd}(k, s)=1$. Then the action of $\left(e^{\frac{2 \pi \imath l}{r}}, e^{\frac{2 \pi \imath k}{s}}\right)$ on $(u, v)$ becomes $(u, v) \longmapsto$ $\left(e^{\frac{2 \pi \imath}{r s}} u, e^{\frac{2 \pi \imath(-s l-k r)}{r s}} v\right)$. Hence $S^{3} / \phi\left(\mathbb{Z}_{r} \times \mathbb{Z}_{s}\right)$ corresponds to the lens space $L(p, q)$ with $p=r s$ and $q=-l s-k r$.

Notice, though, the following ambiguity in describing the subgroups of $S^{3} \times S^{3}$. We have $\phi\left(\mathbb{Z}_{r} \times \mathbb{Z}_{2 s}\right)=\phi\left(\mathbb{Z}_{2 r} \times \mathbb{Z}_{s}\right)$, in particular $\phi\left(\mathbb{Z}_{r} \times \mathbb{Z}_{2}\right)=\phi\left(\mathbb{Z}_{2 r} \times\{e\}\right)$. A small calculation shows that the inhomogeneous lens spaces correspond to $\mathbb{Z}_{r} \times \mathbb{Z}_{s}$ with $\operatorname{gcd}(r, s)=1$ and $r \geq 3, s \geq 3$.

Remark. Note that the above described example is a special class of lens spaces, namely those which correspond to $S^{3} / \phi\left(\mathbb{Z}_{r} \times \mathbb{Z}_{s}\right)$ with $\operatorname{gcd}(r, s)=1$. In general a lens space always corresponds to $S^{3} / G$ where $G$ is a cyclic subgroup of $\mathbb{Z}_{r} \times \mathbb{Z}_{s}$ with $r \neq 1$ and $s \neq 1$ not necessarily relatively prime.

To describe Scott's classification consider the homomorphism

$$
\begin{aligned}
\pi: S O(4) & \longrightarrow S O(3) \times S O(3) \\
x \mapsto q_{1} x q_{2}^{-1} & \longmapsto\left(x \mapsto q_{1} x q_{1}^{-1}, x \mapsto q_{2} x q_{2}^{-1}\right)
\end{aligned}
$$

with kernel $K=\{\mathrm{Id},-\mathrm{Id}\}$. We will use the following lemma:

Lemma 2.1. Let $\pi: S O(4) \longrightarrow S O(3) \times S O(3)$. If a finite subgroup $G$ of $S O(4)$ acts freely on $S^{3}$, then $H=\pi(G)$ acts freely on $S^{3} /\{ \pm I d\}=\mathbb{R} P^{3}$. Conversely, if $H \subset S O(3) \times S O(3)$ acts freely on $\mathbb{R} P^{3}$ then $G=\pi^{-1}(H)$ acts freely on $S^{3}$.

Proof. For the forward direction see the proof by Scott ([S], Lemma 4.8). Conversely, let $H$ act freely on $\mathbb{R} P^{3}$ and $G=\pi^{-1}(H)$. If $g x=x$ in $S^{3}$, then 
$\pi(g)[x]=[x]$ for $[x] \in \mathbb{R} P^{3}$. Therefore $g=\operatorname{Id}$ or $g=-\mathrm{Id}$ and hence $\pi(g)=\mathrm{Id}$, which implies that $G$ acts freely on $S^{3}$.

Now we consider the possibilities for a finite subgroup $H$ of $S O(3) \times S O(3)$ acting freely on $\mathbb{R} P^{3}$. Let $H_{1}$ and $H_{2}$ denote the projections of $H$ into the two factors of $S O(3) \times S O(3)$. Hence $H$ is a subgroup of $H_{1} \times H_{2}$. Recall that a finite subgroup of $S O(3)$ is cyclic, dihedral or the orientation preserving symmetry group of a regular solid. These are:

1. the cyclic groups $\mathbb{Z}_{m}, m \geq 1$ of order $m$,

2. the dihedral groups $D_{m}, m \geq 2$ of order $2 m$,

3 . the tetrahedral group $T$ of order 12 ,

4. the octahedral group $O$ of order 24 ,

5. the icosahedral group $I$ of order 60 .

Note that $T$ is isomorphic to the alternating group on four elements $A_{4}, O$ is isomorphic to the symmetric group on four elements $S_{4}$, and $I$ is isomorphic to $A_{5}$. Also note that all these groups have even order apart from the obvious cyclic groups. In general a subgroup of a direct product need not itself be a direct product. We will need the following:

Definition 2.2. Let $G$ be any group, let $H$ be a finite subgroup of $G \times G$ and let $H_{1}$ and $H_{2}$ denote the projections of $H$ into the two factors of $G \times G$. Then $H$ is called a diagonal subgroup of $H_{1} \times H_{2}$ if there are surjections $f_{1}$ and $f_{2}$ from $H_{1}$ and $H_{2}$ respectively to some group $A$, and $H$ is the kernel of the map $H_{1} \times H_{2} \longrightarrow A$ whose restriction to $H_{i}$ is $f_{i}$.

In other words we have the following exact sequence:

$$
1 \longrightarrow H \longrightarrow H_{1} \times H_{2} \stackrel{f}{\longrightarrow} A \longrightarrow 1
$$

where $f \mid H_{i}=f_{i}$.

Now we have all the preparations to state the classification theorem:

Theorem 2.3. $[\mathrm{S}]$ Let $H$ be a finite subgroup of $S O(3) \times S O(3)$ which acts freely on $S^{3} /\{ \pm I d\}$, and let $H_{1}$ and $H_{2}$ denote the projections of $H$ into the two factors.

Then $H$ is one of the following subgroups:

(i) $H=\mathbb{Z}_{p} \times H_{2}$ with $\left(p,\left|H_{2}\right|\right)=1, p \neq 1$ and $H_{2} \in\left\{D_{n}, T, O, I\right\}$;

(ii) $H$ is a diagonal subgroup of index three in $\mathbb{Z}_{3 n} \times T$ with $n$ odd;

(iii) $H$ is a diagonal subgroup of index two in $\mathbb{Z}_{2 m} \times D_{n}$ with $n$ odd and $\operatorname{gcd}(n, m)=$ 1

(iv) $H$ is a cyclic subgroup of $\mathbb{Z}_{r} \times \mathbb{Z}_{s}$ with $r \neq 1, s \neq 1$;

(v) $H=\{e\} \times H_{2}$ with $\mathrm{H}_{2}$ any finite subgroup of $\mathrm{SO}(3)$.

Notice that case (v) corresponds precisely to the homogeneous spherical space forms. Theorem 2.3 clearly induces a classification of the corresponding subgroup $G \subset S O(4)$ as follows. If $G$ has even order, then there exists an element $\gamma$ of $G$ with $\gamma^{2}=\mathrm{Id}$ and since $G$ acts freely, $\gamma=-\mathrm{Id}$. Hence $\operatorname{Ker}(\pi) \subset G$, which means $G=\pi^{-1}(H)$ with $H=\pi(G)$ and $\mathbb{R} P^{3} / H \cong S^{3} / \pi^{-1}(H) \cong S^{3} / G$. If $G$ has odd order, then $\pi: G \longrightarrow \pi(G)=H$ is an isomorphism implying that $H$ has also odd order. Hence we are in case (iv) or (v) of Theorem 2.3, which means that $H$, and hence $G$, must be a cyclic group of odd order. 
Hence our space forms $S^{3} / G$ are either lens spaces $L(p, q)$ with $p$ odd (which are not covered by $\mathbb{R} P^{3}$ ) or of the form $S^{3} / G \cong \mathbb{R} P^{3} / H$ with $H$ as in Theorem 2.3 .

\section{Proof of Theorem 1}

We want to show that, except for cases (1) and (2) of Theorem 1, for each inhomogeneous space form $S^{3} / G$ there exists an inhomogeneous lens space covering it.

If $U$ is a subgroup of $G$, then $\pi: S^{3} / U \longrightarrow S^{3} / G$ is a covering. Therefore we need to show that for each such $G$ there exist a $\mathbb{Z}_{r} \times \mathbb{Z}_{s} \subset S^{3} \times S^{3}$ with $r \geq 3$ and $s \geq 3$ such that $\phi\left(\mathbb{Z}_{r} \times \mathbb{Z}_{s}\right) \subset G$. As we saw in the discussion after Theorem 2.3 , if $S^{3} / G$ is not a lens space, then $S^{3} / G=\mathbb{R} P^{3} / H$ and hence it suffices to examine the cases (i) to (ii) in Theorem 2.3 and to show that there exists in each case a $\mathbb{Z}_{r} \times \mathbb{Z}_{s} \subset S O(3) \times S O(3)$ with $r \neq 1$ and $s \neq 1$ and $\mathbb{Z}_{r} \times \mathbb{Z}_{s} \subset H$. This can be done easily.

Let us start with the first case of Theorem 2.3, where $H=\mathbb{Z}_{p} \times H_{2}$ with $\operatorname{gcd}\left(p,\left|H_{2}\right|\right)=1, p \neq 1$ and $H_{2} \in\left\{D_{n}, T, O, I\right\}$. Since $D_{n}, T, O$ and $I$ all have even order, each has a subgroup of order 2. Hence $H$ admits the subgroup $\mathbb{Z}_{p} \times \mathbb{Z}_{2}$ with $\operatorname{gcd}(p, 2)=1$ and $p \neq 1$.

In the second case we have the following exact sequence:

$$
1 \longrightarrow H \longrightarrow \mathbb{Z}_{3 n} \times T \longrightarrow \mathbb{Z}_{3} \longrightarrow 1,
$$

with $n$ odd. Hence $H$ is the kernel of the surjective homomorphism $\tau: \mathbb{Z}_{3 n} \times T \longrightarrow$ $\mathbb{Z}_{3}$. Since $\mathbb{Z}_{3}$ has no non-trivial elements of order 2 , we have that

$$
\mathbb{Z}_{2} \times \mathbb{Z}_{2} \cong\left\{(0, t) \mid t \in T, t^{2}=e\right\} \subset \operatorname{Ker}(\tau) .
$$

On the other hand, if $n>1$, we also obtain that

$$
\mathbb{Z}_{n} \cong\left\{\left(z^{3}, e\right) \mid z \in \mathbb{Z}_{3 n}\right\} \subset \operatorname{Ker}(\tau) .
$$

Hence $\mathbb{Z}_{n} \times\left(\mathbb{Z}_{2} \times \mathbb{Z}_{2}\right) \subset \operatorname{Ker}(\tau)=H$ and in particular there exists $\mathbb{Z}_{n} \times \mathbb{Z}_{2} \subset H$ with $\operatorname{gcd}(n, 2)=1$ and $n>1$.

In the third case we have the following exact sequence:

$$
1 \longrightarrow H \longrightarrow \mathbb{Z}_{2 m} \times D_{n} \longrightarrow \mathbb{Z}_{2} \longrightarrow 1
$$

where $\operatorname{gcd}(m, n)=1$ and $n$ odd. Hence $H$ is the kernel of the surjective homomorphism $\tau: \mathbb{Z}_{2 m} \times D_{n} \longrightarrow \mathbb{Z}_{2}$. Since the image of every element in $\mathbb{Z}_{2 m} \times D_{n}$ obviously squares to be the identity in $\mathbb{Z}_{2}$, for $m>1$, we have that $\mathbb{Z}_{m} \cong$ $\left\{\left(z^{2}, e\right) \mid z \in \mathbb{Z}_{2 m}\right\} \subset \operatorname{Ker}(\tau)$, and $\mathbb{Z}_{n} \cong\left\{(0, d) \mid d \in D_{n}, d^{n}=e\right\} \subset \operatorname{Ker}(\tau)$. Hence $\mathbb{Z}_{n} \times \mathbb{Z}_{m} \subset \operatorname{Ker}(\tau)=H$ with $\operatorname{gcd}(m, n)=1$ with $m>1$ and $n>1$.

Thus we have shown that, with the exception of $n=1$ in case (ii) and $p=1$ in case (iii), in each of the cases of Theorem 2.3 there exist cyclic groups $\mathbb{Z}_{r}$ and $\mathbb{Z}_{s}$ with $r \neq 1$ and $s \neq 1$ such that $\mathbb{Z}_{r} \times \mathbb{Z}_{s} \subset H$.

For $n=1$ in case (ii), one can show that $H$ is isomorphic to $T$. Hence this case corresponds exactly to case (1) of Theorem 1 where, following $[\mathrm{S}], \tilde{H}$ denotes the pre-image of $H$ in $S^{3}$ under the natural homomorphism $\psi: S^{3} \longrightarrow S O(3)$. As $H \cong T$ is a diagonal subgroup of $\mathbb{Z}_{3} \times T$ of index three, it follows that there are no subgroups of the form $\mathbb{Z}_{r} \times \mathbb{Z}_{s}$ of $H$ with $r \neq 1$ and $s \neq 1$.

For $p=1$ in case (iii), one can show that $H$ is isomorphic to $D_{n}$. Hence this case corresponds exactly to case (2) of Theorem 1 . As $H \cong D_{n}$ is a diagonal 
subgroup of $\mathbb{Z}_{2} \times D_{n}$ of index two, it follows that there are no subgroups of the form $\mathbb{Z}_{r} \times \mathbb{Z}_{s}$ of $H$ with $r \neq 1$ and $s \neq 1$.

This finishes the proof of Theorem 1 .

Remark. Theorem 1 also holds in the homogeneous setting, i.e. every homogeneous spherical space form can be covered by a homogeneous lens space. In fact, as all non-cyclic subgroups of $S O(3)$ are of even order, we can always cover homogeneous spherical space forms by $L(2,1)$.

\section{Proof of Theorem 2}

\subsection{Preliminaries.}

In order to minimally isometrically immerse any space form into a sphere we need the components of the immersion to consist of harmonic homogeneous polynomials of a given degree in four real variables. This follows from the previously quoted theorem by Takahashi. In our case $M=S^{3}$, and the eigenfunctions of $S^{3}$ are harmonic homogeneous polynomials in four real variables. The isometry condition translates into the following system of partial differential equations:

$$
\sum_{k=1}^{n} \frac{\partial f_{k}}{\partial x_{i}} \frac{\partial f_{k}}{\partial x_{j}}=\delta_{i j}\left(\sum_{l=1}^{4} x_{l}^{2}\right)^{g-1}+C x_{i} x_{j}\left(\sum_{l=1}^{4} x_{l}^{2}\right)^{g-2} .
$$

Here the $f_{k}$ denote the components of $f, g$ stands for the degree of the polynomials used and $C$ is the constant $C=\frac{3 g}{g+2}-1$.

A slightly different system of differential equations was used by N. Wallach [Wa]. He assumed the target sphere to have radius 1. Equations (4.1.0) can be derived in the following way: Let $f: S^{n}(1) \hookrightarrow S^{N}\left(\frac{1}{\sqrt{K}}\right)$ be a minimal isometric immersion. Here $K=\frac{\lambda}{n}$ and $\lambda$ is an eigenvalue of the Laplacian on $S^{n}(1)$. Let $x_{1}, \ldots, x_{n+1}$ be the standard coordinates of $\mathbb{R}^{n+1}$. Then the metric $\mathfrak{g}$ on $S^{n}(1)$ is given by $\mathfrak{g}=\sum_{i=1}^{n+1} d x_{i} \otimes d x_{i}$. The isometry condition translates into $\mathfrak{g}=\sum_{i=1}^{N+1} d f_{i} \otimes d f_{i}$. Let $\mathbb{R}^{+}=\{t \in \mathbb{R} \mid t<0\}$ and let $\phi: \mathbb{R}^{+} \times S^{n}(1) \hookrightarrow \mathbb{R}^{n+1},(t, x) \mapsto t x$. Note that for harmonic homogeneous polynomials $\mathfrak{p}$ of degree $g$ we have that $\mathfrak{p}(t x)=t^{g} \mathfrak{p}(x)$ and therefore $f_{i}(t x)=t^{g} f_{i}(x)$. Explicit computations of the differential $\phi^{*}$ of $\phi$ yield

$$
\begin{aligned}
\phi^{*}\left(\sum_{i=1}^{n+1} d x_{i} \otimes d x_{i}\right)_{(t, x)} & =t^{2} \mathfrak{g}_{x}+(d t \otimes d t)_{t} \\
\phi^{*}\left(\sum_{i=1}^{N+1} d f_{i} \otimes d f_{i}\right)_{(t, x)} & =\frac{1}{K} g^{2} t^{2 g-2}(d t \otimes d t)_{t}+t^{2 g}\left(\sum_{i=1}^{N+1} d f_{i} \otimes d f_{i}\right)_{x} \\
& =\frac{1}{K} g^{2} t^{2 g-2}(d t \otimes d t)_{t}+t^{2 g} \mathfrak{g}_{x} \\
& =t^{2 g-2}\left(\frac{g^{2}}{K}-1\right)(d t \otimes d t)_{t}+t^{2 g-2}\left((d t \otimes d t)_{t}+t^{2} \mathfrak{g}_{x}\right) \\
& =t^{2 g-2}\left(\frac{g^{2}}{K}-1\right)(d t \otimes d t)_{t}+t^{2 g-2} \phi^{*}\left(\sum_{i=1}^{n+1} d x_{i} \otimes d x_{i}\right)_{(t, x)}
\end{aligned}
$$


Setting $r(x)=\sum_{i=1}^{n+1} x_{i}^{2}$ for $x \in \mathbb{R}^{n+1}$, we have

$$
\left(\phi^{-1}\right)^{*}(d t)_{x}=r^{-\frac{1}{2}} \sum_{i=1}^{n+1} x_{i} d x_{i}
$$

Combining this with the above equations yields

$$
\sum_{i=1}^{N+1} d f_{i} \otimes d f_{i}=r^{g-1} \sum_{i=1}^{n+1} d x_{i} \otimes d x_{i}+\left(\frac{g^{2}}{K}-1\right) r^{g-2} \sum_{i, j=1}^{n+1} x_{i} x_{j} d x_{i} \otimes d x_{j} .
$$

This equation is equivalent to the system of partial differential equations

$$
\sum_{k=1}^{N+1} \frac{\partial f_{k}}{\partial x_{i}} \frac{\partial f_{k}}{\partial x_{j}}=\delta_{i j}\left(\sum_{l=1}^{n+1} x_{l}^{2}\right)^{g-1}+\left(\frac{g^{2}}{K}-1\right) x_{i} x_{j}\left(\sum_{l=1}^{n+1} x_{l}^{2}\right)^{g-2} .
$$

Observing that for $n=3$ we have that $K=\frac{\lambda}{3}$ and $\lambda=g(g+2)$, we obtain equations (4.1.0).

In this setting it is more natural to translate the system (4.1.0) of four real variables into a system of two complex variables. To do so we will set $z=x_{1}+$ $\imath x_{2}, w=x_{3}+\imath x_{4}$ and hence

$$
\begin{aligned}
& \frac{\partial f}{\partial x_{1}}=\frac{\partial f}{\partial z}+\frac{\partial f}{\partial \bar{z}} ; \quad \frac{\partial f}{\partial x_{2}}=\frac{1}{\imath}\left(\frac{\partial f}{\partial z}-\frac{\partial f}{\partial \bar{z}}\right) \\
& \frac{\partial f}{\partial x_{3}}=\frac{\partial f}{\partial w}+\frac{\partial f}{\partial \bar{w}} ; \quad \frac{\partial f}{\partial x_{4}}=\frac{1}{\imath}\left(\frac{\partial f}{\partial w}-\frac{\partial f}{\partial \bar{w}}\right) \\
& \Delta f=4\left(\frac{\partial^{2} f}{\partial z \partial \bar{z}}+\frac{\partial^{2} f}{\partial w \partial \bar{w}}\right) .
\end{aligned}
$$

Using these rules we obtain the following six equations:

$$
\begin{aligned}
& \sum_{k=1}^{N+1}\left(\frac{\partial f_{k}}{\partial z}\right)^{2}=\frac{C}{4}(z \bar{z}+w \bar{w})^{g-2} \bar{z}^{2} \\
& \sum_{k=1}^{N+1}\left(\frac{\partial f_{k}}{\partial w}\right)^{2}=\frac{C}{4}(z \bar{z}+w \bar{w})^{g-2} \bar{w}^{2} \\
& \sum_{k=1}^{N+1} \frac{\partial f_{k}}{\partial z} \frac{\partial f_{k}}{\partial \bar{z}}=\frac{1}{2}(z \bar{z}+w \bar{w})^{g-1}+\frac{C}{4}(z \bar{z}+w \bar{w})^{g-2} z \bar{z} \\
& \sum_{k=1}^{N+1} \frac{\partial f_{k}}{\partial w} \frac{\partial f_{k}}{\partial \bar{w}}=\frac{1}{2}(z \bar{z}+w \bar{w})^{g-1}+\frac{C}{4}(z \bar{z}+w \bar{w})^{g-2} w \bar{w} \\
& \sum_{k=1}^{N+1} \frac{\partial f_{k}}{\partial z} \frac{\partial f_{k}}{\partial w}=\frac{C}{4}(z \bar{z}+w \bar{w})^{g-2} \bar{z} \bar{w} \\
& \sum_{k=1}^{N+1} \frac{\partial f_{k}}{\partial \bar{z}} \frac{\partial f_{k}}{\partial w}=\frac{C}{4}(z \bar{z}+w \bar{w})^{g-2} z \bar{w}
\end{aligned}
$$


In our case we want to construct a minimal isometric immersion of an inhomogeneous lens space into a sphere. Therefore we use the above mentioned construction but additionally require the harmonic polynomials to be invariant under the corresponding group action.

\subsection{Description of the group action.}

Consider the following action of $G \cong \mathbb{Z}_{p}$ on $S^{3}$. Let $\omega=e^{\frac{2 \pi}{p} \imath}$ be a generator of $\mathbb{Z}_{p}$. Then the group action is given by $\omega \cdot(z, w)=\left(e^{\frac{2 \pi}{p} \imath} z, e^{\frac{2 \pi q}{p} \imath} w\right)$ and the resulting quotient space $S^{3} / G$ is the lens space $L(p, q)$. In the above complex notation the action of $\mathbb{Z}_{p}$ on $\mathfrak{p} \in \mathbb{C}[z, w]$ is given by

$$
\begin{aligned}
\omega \cdot \mathfrak{p}(z, w, \bar{z}, \bar{w}) & =\mathfrak{p}\left(\omega z, \omega^{q} w, \bar{\omega} \bar{z}, \bar{\omega}^{q} \bar{w}\right) \\
& =\mathfrak{p}\left(e^{\frac{2 \pi}{p} \imath} z, e^{\frac{2 \pi q}{p} \imath} w, e^{-\frac{2 \pi}{p} \imath} \bar{z}, e^{-\frac{2 \pi q}{p} \imath} \bar{w}\right) .
\end{aligned}
$$

Hence in order for a monomial $z^{a} w^{b} \bar{z}^{c} \bar{w}^{d}$ to be invariant under this action, we need that $p$ divides $a+q b-c-q d$. Moreover, a set of polynomials is invariant under this particular action if and only if the corresponding set of monomials is invariant under the action.

Conclusion. A set of harmonic homogeneous polynomials is invariant under the above described action if and only if all generating monomials $z^{a} w^{b} \bar{z}^{c} \bar{w}^{d}$ satisfy the condition:

$$
a+q b-c-q d \equiv 0 \quad \bmod p .
$$

\subsection{Analyzing the isometry condition.}

We will use the following isometry of $S^{3}$ :

Definition 4.3.1. Denote by $T$ the involution $T: S^{3} \rightarrow S^{3}, T(z, w, \bar{z}, \bar{w})=$ $(\bar{z}, w, z, \bar{w})$.

Remark. The reason we chose this particular isometry is that it is an isometry of $S^{3}$ which never covers an isometry of an inhomogeneous lens space $L(p, q)$ for any $p$ and any $q$ with $\operatorname{gcd}(p, q)=1$ and $q \not \equiv \pm 1 \bmod p$. This choice will simplify the calculations in section 4.4 considerably.

Note that $T$ commutes with multiplication and partial derivatives in the following way:

Lemma 4.3.2. Let $T$ be defined as above and let $m, m_{1}$ and $m_{2}$ be monomials, all of the same fixed degree. Then the following properties hold:

$$
\begin{aligned}
T\left(m_{1} \cdot m_{2}\right) & =T\left(m_{1}\right) \cdot T\left(m_{2}\right) \quad \text { and } \\
T\left(\frac{\partial m}{\partial z}\right) & =\frac{\partial}{\partial \bar{z}}[T(m)], T\left(\frac{\partial m}{\partial w}\right)=\frac{\partial}{\partial w}[T(m)], \\
T\left(\frac{\partial m}{\partial \bar{z}}\right) & =\frac{\partial}{\partial z}[T(m)], T\left(\frac{\partial m}{\partial \bar{w}}\right)=\frac{\partial}{\partial \bar{w}}[T(m)] .
\end{aligned}
$$

Proof. This is a straightforward computation.

Denote by $R_{i}$ the right hand side of (4.1.i) for each $i \in\{1, \ldots, 6\}$. Observe that $T\left(R_{1}\right)=\bar{R}_{1}, T\left(R_{2}\right)=R_{2}, T\left(R_{3}\right)=R_{3}, T\left(R_{4}\right)=R_{4}, T\left(R_{5}\right)=R_{6}, T\left(R_{6}\right)=R_{5}$. 
Using Lemma 4.3.2 and the fact that $T^{2}=I d$, equations (4.1.1) to (4.1.6) translate into the following system:

$$
\begin{aligned}
& \sum_{k=1}^{n}\left(\frac{\partial T\left(f_{k}\right)}{\partial z}\right)^{2}=\sum_{k=1}^{n}\left(\frac{\partial f_{k}}{\partial z}\right)^{2}=R_{1}, \\
& \sum_{k=1}^{n}\left(\frac{\partial T\left(f_{k}\right)}{\partial w}\right)^{2}=\sum_{k=1}^{n}\left(\frac{\partial f_{k}}{\partial w}\right)^{2}=R_{2}, \\
& \sum_{k=1}^{n} \frac{\partial T\left(f_{k}\right)}{\partial z} \frac{\partial T\left(f_{k}\right)}{\partial \bar{z}}=\sum_{k=1}^{n} \frac{\partial f_{k}}{\partial z} \frac{\partial f_{k}}{\partial \bar{z}}=R_{3}, \\
& \sum_{k=1}^{n} \frac{\partial T\left(f_{k}\right)}{\partial w} \frac{\partial T\left(f_{k}\right)}{\partial \bar{w}}=\sum_{k=1}^{n} \frac{\partial f_{k}}{\partial w} \frac{\partial f_{k}}{\partial \bar{w}}=R_{4}, \\
& \sum_{k=1}^{n} \frac{\partial T\left(f_{k}\right)}{\partial z} \frac{\partial T\left(f_{k}\right)}{\partial w}=\sum_{k=1}^{n} \frac{\partial f_{k}}{\partial z} \frac{\partial f_{k}}{\partial w}=R_{5}, \\
& \sum_{k=1}^{n} \frac{\partial T\left(f_{k}\right)}{\partial \bar{z}} \frac{\partial T\left(f_{k}\right)}{\partial w}=\sum_{k=1}^{n} \frac{\partial f_{k}}{\partial \bar{z}} \frac{\partial f_{k}}{\partial w}=R_{6} .
\end{aligned}
$$

\section{Definition 4.3.4.}

$$
\begin{aligned}
M^{g}:= & \left\{m_{i} \mid m_{i} \text { all monomials in two complex variables of degree } g\right\} \\
= & \left\{z^{a} w^{b} \bar{z}^{c} \bar{w}^{d} \mid a+b+c+d=g\right\}, \\
M_{G}^{g}:= & \left\{m_{i} \mid m_{i} \text { all monomials of degree } g\right. \text { invariant under action of } \\
& \left.G=\mathbb{Z}_{p} \text { as in } 4.2\right\} \\
= & \left\{z^{a} w^{b} \bar{z}^{c} \bar{w}^{d} \mid a+b+c+d=g, a+b q-c-d q \equiv 0 \text { mod } p\right\}, \\
P^{g}:= & \left\{\mathfrak{p}_{i} \mid \mathfrak{p}_{i} \text { a harmonic homogeneous polynomial of degree } g,\right. \\
& \left.i=1, \cdots,(g+1)^{2}\right\}, \\
P_{G}^{g}:= & \left\{\mathfrak{p}_{i} \mid \mathfrak{p}_{i} \text { a harmonic homogeneous polynomial of degree } g\right. \\
& \left.\quad \text { invariant under the action of } G=\mathbb{Z}_{p}, i=1, \cdots, m\right\}, \\
Q^{g}:= & \left\{\mathfrak{q}_{i} \mid \mathfrak{q}_{i} \in P^{g} \text { such that } T\left(\mathfrak{q}_{i}\right)=\mathfrak{q}_{i} \text { or } T\left(\mathfrak{q}_{i}\right)=\overline{\mathfrak{q}}_{i}\right\}, \\
Q_{G}^{g}:= & Q^{g} \cap P_{G}^{g}, \\
T\left(P_{G}^{g}\right):= & \left\{T\left(\mathfrak{p}_{i}\right) \mid \mathfrak{p}_{i} \in P_{G}^{g}\right\} .
\end{aligned}
$$

Lemma 4.3.5. One can always choose a basis $\left\{\mathfrak{p}_{i}, i=1, \cdots,(g+1)^{2}\right\}$ of $P^{g}$ such that $T\left(\mathfrak{p}_{i}\right)=\mathfrak{p}_{j}$ for some $j \in\left\{1, \cdots,(g+1)^{2}\right\}$.

Proof. Construct a basis of all harmonic homogeneous polynomials of a given degree $g$ in the following way: Take $m_{1}=z^{a} w^{b} \bar{z}^{c} \bar{w}^{d} \in M^{g}$. Then

$$
\begin{aligned}
\mathfrak{p}_{1}=z^{a} w^{b} \bar{z}^{c} \bar{w}^{d} & \\
+\sum_{i=0}^{N}(-1)^{i+1}\{ & \prod_{k=0}^{i} \frac{(a-k)(c-k)}{(b+k+1)(d+k+1)} z^{a-i-1} w^{b+i+1} \bar{z}^{c-i-1} \bar{w}^{d+i+1} \\
& \left.+\prod_{k=0}^{i} \frac{(b-k)(d-k)}{(a+k+1)(c+k+1)} z^{a+i+1} w^{b-i-1} \bar{z}^{c+i+1} \bar{w}^{d-i-1}\right\}
\end{aligned}
$$


is a harmonic homogeneous polynomial, where $N=\max (\min (a, c), \min (b, d))-1$.

Let $M_{1}^{g}=\left\{\right.$ all monomials used in the construction of $\left.\mathfrak{p}_{1}\right\}$. Then let $M^{g}=$ $M^{g} \backslash M_{1}^{g}$ and repeat the above step until all of $M^{g}$ is exhausted. In this construction each polynomial $\mathfrak{p}_{i}$ is built with different monomials, hence all $\mathfrak{p}_{i}$ are linearly independent. Also, each $\mathfrak{p}_{i}$ is unique up to scalar multiples, i.e. no other combination of monomials $m_{j} \in M_{j}^{g}$ can be used to construct $\mathfrak{p}_{i}$. Therefore the above constructed $\mathfrak{p}_{i}$ form a basis for $P^{g}$. Note that this basis has the property that $a-c$ and $b-d$ is constant for all monomials $z^{a} w^{b} \bar{z}^{c} \bar{w}^{d}$ in a given polynomial. Consider the polynomial $\mathfrak{p}_{1} \in P^{g}$ as constructed above. One has

$$
\begin{aligned}
T\left(\mathfrak{p}_{1}\right)= & z^{c} w^{b} \bar{z}^{a} \bar{w}^{d} \\
+\sum_{i=0}^{N}(-1)^{i+1}\{ & \prod_{k=0}^{i} \frac{(a-k)(c-k)}{(b+k+1)(d+k+1)} z^{c-i-1} w^{b+i+1} \bar{z}^{a-i-1} \bar{w}^{d+i+1} \\
& \left.+\prod_{k=0}^{i} \frac{(b-k)(d-k)}{(a+k+1)(c+k+1)} z^{c+i+1} w^{b-i-1} \bar{z}^{a+i+1} \bar{w}^{d-i-1}\right\} .
\end{aligned}
$$

We need to find some $\mathfrak{p}_{j} \in P^{g}$ such that $T\left(\mathfrak{p}_{1}\right)=\mathfrak{p}_{j}$. We start with the monomial $m=z^{c} w^{b} \bar{z}^{a} \bar{w}^{d}$. Then

$$
\begin{aligned}
\mathfrak{p}_{2} & =z^{c} w^{b} \bar{z}^{a} \bar{w}^{d} \\
& +\sum_{i=0}^{N}(-1)^{i+1}\left\{\prod_{k=0}^{i} \frac{(b-k)(d-k)}{(a+k+1)(c+k+1)} z^{c-i-1} w^{b+i+1} \bar{z}^{a-i-1} \bar{w}^{d+i+1}\right. \\
& \left.\quad+\prod_{k=0}^{i} \frac{(a-k)(c-k)}{(b+k+1)(d+k+1)} z^{c+i+1} w^{b-i-1} \bar{z}^{a+i+1} \bar{w}^{d-i-1}\right\} \\
& =T\left(\mathfrak{p}_{1}\right) .
\end{aligned}
$$

As the choice of $\mathfrak{p}_{1}$ was arbitrary, the basis $\mathfrak{p}_{i}$ has the property that given $\mathfrak{p}_{i} \in P^{g}$, there exists a $j \in\left\{1, \cdots,(g+1)^{2}\right\}$ such that $T\left(\mathfrak{p}_{i}\right)=\mathfrak{p}_{j}$.

Remark. As the referee has kindly pointed out, the above constructed basis is up to a constant multiple a basis obtained through harmonic projection of the monomials, as described in $[\mathrm{V}]$.

Using Lemma 4.3.5 the following construction is well defined:

\section{Lemma 4.3.6.}

(i) $g$ even: One can express $f_{k}$ as the following sum:

$$
f_{k}=\sum_{i=1}^{\left(\frac{g}{2}\right)^{2}} a_{k i} \mathfrak{p}_{i}+\bar{a}_{k i} \overline{\mathfrak{p}}_{i}+b_{k i} T\left(\mathfrak{p}_{i}\right)+\bar{b}_{k i} T\left(\overline{\mathfrak{p}}_{i}\right)+\sum_{i=1}^{g} c_{k i} \mathfrak{q}_{i}+\bar{c}_{k i} \overline{\mathfrak{q}}_{i}+d_{k 1} \mathfrak{r}_{1},
$$

where $\mathfrak{p}_{i} \in P^{g}, \mathfrak{q}_{i}, \mathfrak{r}_{1} \in Q^{g}$ and $\mathfrak{r}_{1}=\overline{\mathfrak{r}}_{1}$

(ii) $g$ odd: One can express $f_{k}$ as

$$
f_{k}=\sum_{i=1}^{\frac{g^{2}-1}{4}} a_{k i} \mathfrak{p}_{i}+\bar{a}_{k i} \overline{\mathfrak{p}}_{i}+b_{k i} T\left(\mathfrak{p}_{i}\right)+\bar{b}_{k i} T\left(\overline{\mathfrak{p}}_{i}\right)+\sum_{i=1}^{g+1} c_{k i} \mathfrak{q}_{i}+\bar{c}_{k i} \overline{\mathfrak{q}}_{i},
$$

where $\mathfrak{p}_{i} \in P^{g}, \mathfrak{q}_{i} \in Q^{g}$. 
Proof. Our goal is to show that we can use a permutation of the basis constructed in Lemma 4.3.5 to express $f_{k}$ as claimed.

(i) Assume $g$ is even. Using the basis for $P^{g}$ as constructed in Lemma 4.3.5 we need to find those polynomials

$$
\begin{aligned}
\mathfrak{p} & =z^{a} w^{b} \bar{z}^{c} \bar{w}^{d} \\
+\sum_{i=0}^{N}(-1)^{i+1}\{ & \prod_{k=0}^{i} \frac{(a-k)(c-k)}{(b+k+1)(d+k+1)} z^{a-i-1} w^{b+i+1} \bar{z}^{c-i-1} \bar{w}^{d+i+1} \\
& \left.+\prod_{k=0}^{i} \frac{(b-k)(d-k)}{(a+k+1)(c+k+1)} z^{a+i+1} w^{b-i-1} \bar{z}^{c+i+1} \bar{w}^{d-i-1}\right\}
\end{aligned}
$$

for which $T(\mathfrak{p})=\mathfrak{p}$ or $T(\mathfrak{p})=\overline{\mathfrak{p}}$ holds.

Consider first the case of $T(\mathfrak{p})=\mathfrak{p}$. From the construction it follows that then $a$ must be equal to $c$. Hence if $T(\mathfrak{p})=\mathfrak{p}$, then all monomials $z^{a} w^{b} \bar{z}^{c} \bar{w}^{d} \in \mathfrak{p}$ have the property that $a=c$. A counting argument shows that there are exactly $g+1$ such polynomials: The chosen basis has the property that $a-c$ and $b-d$ is constant for all monomials $z^{a} w^{b} \bar{z}^{c} \bar{w}^{d}$ in a given polynomial. Also note that $|(a-c)+(b-d)|=|g-2(c+d)|$ is even (resp. odd) if $g$ is even (resp. odd) and that $|(a-c)+(b-d)| \leq g$. Hence we can represent the polynomials of our fixed basis as tuples $(a-c, b-d) \in \mathbb{Z} \times \mathbb{Z}$. In our case $a-c=0$ and we are looking for all possible polynomials of the form $(0, b-d)$. As $(b-d)$ can be any even number of absolute value less or equal to $g$, we obtain $g+1$ possibilities. Here the case of $(0,0)$ corresponds to the real polynomial $\mathfrak{r}_{1}$ as $a=c$ and $b=d$.

Next, consider the case of $T(\mathfrak{p})=\overline{\mathfrak{p}}$. From the construction it follows that then $b$ must be equal to $d$. Following the argument of the previous case we are now looking for all possible polynomials of the form $(a-c, 0)$. Again $(a-c)$ can be any even number of absolute value less or equal to $g$ and we obtain $g$ possibilities excluding the case of $(0,0)$ which was already covered in the previous case. Note that the counting argument above includes all conjugate polynomials $\overline{\mathfrak{p}}_{i}$. Hence we obtain a total of $2 g$ polynomials $\mathfrak{p}_{i}$ with the property that $T\left(\mathfrak{p}_{i}\right)=\mathfrak{p}_{i}$ or $T\left(\mathfrak{p}_{i}\right)=\overline{\mathfrak{p}}_{i}$ and one real polynomial $\mathfrak{r}_{1}$. Since these include all conjugates, the number of remaining polynomials are: $(g+1)^{2}-2 g-1=g^{2}$. When we group these as $\left\{\mathfrak{p}_{i}, T\left(\mathfrak{p}_{i}\right), \overline{\mathfrak{p}}_{i}, T\left(\overline{\mathfrak{p}}_{i}\right)\right\}$, we get exactly $\frac{g^{2}}{4}$ quadruples, each quadruple consisting of a pair $\left\{\mathfrak{p}_{i}, T\left(\mathfrak{p}_{i}\right)\right\}$ and its conjugate. This proves the first part of Lemma 4.3.6.

(ii) Assume $g$ is odd.

In order for a polynomial to be real we need that $a=c$ and $b=d$ for all $z^{a} w^{b} \bar{z}^{c} \bar{w}^{d} \in \mathfrak{p}$. But then $g=a+b+c+d=2 a+2 b$ and the degree $g$ must be even. Hence in the odd degree setting there is no real polynomial. Following a similar counting argument we obtain $2(g+1)$ polynomials $\mathfrak{p}_{i}$ with the property that $T\left(\mathfrak{p}_{i}\right)=\mathfrak{p}_{i}$ or $T\left(\mathfrak{p}_{i}\right)=\overline{\mathfrak{p}}_{i}$. Again the number of remaining quadruples $\left\{\mathfrak{p}_{i}, T\left(\mathfrak{p}_{i}\right), \overline{\mathfrak{p}}_{i}, T\left(\overline{\mathfrak{p}}_{i}\right)\right\}$ is $\frac{g^{2}-1}{4}=\frac{(g+1)^{2}-2 g-2}{4}$.

Remark. As mentioned in the introduction, different choices of bases give rise to congruent immersions. We thus now fix a basis and always express the components $f_{k}$ of the immersion $f$ as in Lemma 4.3.6.

Each of the original set of equations (4.1.1) to (4.1.6) is a polynomial equation which we can solve by equating coefficients. We thus obtain a set of quadratic 
equations in the coefficients $a_{k i}, b_{k i}, c_{k i}, d_{k 1}$. Let

$$
\begin{aligned}
X Y_{i j} & :=\sum_{k=1}^{n} x_{k i} y_{k j} ; X \bar{Y}_{i j}:=\sum_{k=1}^{n} x_{k i} \bar{y}_{k j}, \\
\mathfrak{K} & :=\left\{X Y_{i j}, X \bar{Y}_{i j} \mid X, Y \in\{A, B, C, D\}, i \leq j\right\} .
\end{aligned}
$$

Consider the system of quadratic equations in $a_{k i}, b_{k i}, c_{k i}, d_{k 1}$ as a linear system in elements of $\mathfrak{K}$. This linear system has the property that it splits into an inhomogeneous and a homogeneous system in a natural way.

Lemma 4.3.7. The linear system in elements of $\mathfrak{K}$ splits into an inhomogeneous system in the variables $X \bar{X}_{i i}, X \in\{A, B, C, D\}$ and a homogeneous system in $\mathfrak{K} \backslash\left\{X \bar{X}_{i i}, X \in\{A, B, C, D\}\right\}$.

Proof. We show this splitting property for equation (4.1.1). The proof of the statement for the remaining equations follows the same argument. As we are solving (4.1.1) by coefficient comparison, we need to find monomials $m_{1}=z^{a_{1}} w^{b_{1}} \bar{z}^{c_{1}} \bar{w}^{d_{1}}$ and $m_{2}=z^{a_{2}} w^{b_{2}} \bar{z}^{c_{2}} \bar{w}^{d_{2}}$ such that

$$
\frac{\partial m_{1}}{\partial z} \frac{\partial m_{2}}{\partial z} \in\left\{(z \bar{z}+w \bar{w})^{g-2} \bar{z}^{2}\right\} .
$$

Of course $(*)$ is equivalent to

$$
z^{a_{1}+a_{2}-2} w^{b_{1}+b_{2}} \bar{z}^{c_{1}+c_{2}} \bar{w}^{d_{1}+d_{2}} \in\left\{z^{k} w^{g-2-k} \bar{z}^{k+2} \bar{w}^{g-2-k}, k=0, \cdots g-2\right\} .
$$

We show that

$$
m_{1}, m_{2} \text { satisfy }(*) \Longleftrightarrow m_{1} \in \mathfrak{p} \text { and } m_{2} \in \overline{\mathfrak{p}} \quad \text { for some } \quad \mathfrak{p} \in P^{g} .
$$

This will complete the proof of the lemma as the coefficients of the monomials corresponding to a non-zero right hand side are exactly of the form $X \bar{X}_{i i}, X \in$ $\{A, B, C, D\}$.

$\Leftarrow:$ If $m_{1} \in \mathfrak{p}$ and $m_{2} \in \overline{\mathfrak{p}}$, then $m_{1}=z^{a} w^{b} \bar{z}^{c} \bar{w}^{d}$ and

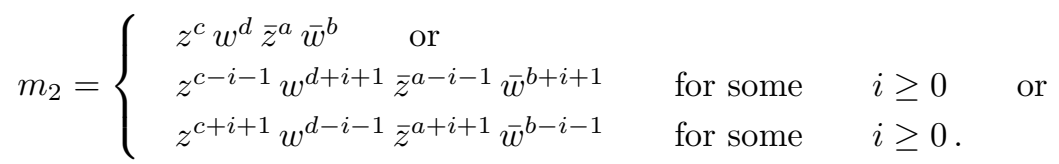

In each case $\frac{\partial m_{1}}{\partial z} \frac{\partial m_{2}}{\partial z}$ is of the form $z^{k} w^{g-2-k} \bar{z}^{k+2} \bar{w}^{g-2-k}$ which implies that $m_{1}$ and $m_{2}$ satisfy $(*)$.

$\Rightarrow$ : Let $m_{1}=z^{a_{1}} w^{b_{1}} \bar{z}^{c_{1}} \bar{w}^{d_{1}}$ and $m_{2}=z^{a_{2}} w^{b_{2}} \bar{z}^{c_{2}} \bar{w}^{d_{2}}$, then

$$
\frac{\partial m_{1}}{\partial z} \frac{\partial m_{2}}{\partial z}=a_{1} a_{2} z^{a_{1}+a_{2}-2} w^{b_{1}+b_{2}} \bar{z}^{c_{1}+c_{2}} \bar{w}^{d_{1}+d_{2}} .
$$

As $m_{1}$ and $m_{2}$ satisfy $(*)$ we obtain $a_{1}+a_{2}=k+2 ; b_{1}+b_{2}=g-k-2 ; c_{1}+c_{2}=$ $k+2 ; d_{1}+d_{2}=g-k-2$. Setting $k+2=l$, these translate to

$$
a_{2}=l-a_{1}, b_{2}=g-l-b_{1}, c_{2}=l-c_{1}, d_{2}=g-l-d_{1} .
$$

Claim. If $z^{a} w^{b} \bar{z}^{c} \bar{w}^{d} \in \mathfrak{p}$, then $z^{l-a} w^{g-l-b} \bar{z}^{l-c} \bar{w}^{g-l-d} \in \overline{\mathfrak{p}}$.

Recall that in our fixed basis of the harmonic homogeneous polynomials (Lemma 4.3.5) a monomial $m_{l}=z^{a_{l}} w^{b_{l}} \bar{z}^{c_{l}} \bar{w}^{d_{l}}$ is in $\overline{\mathfrak{p}}$ if and only if $a_{l}-c_{l}=c-a$ and $b_{l}-d_{l}=d-b$. But the monomial $z^{l-a} w^{g-l-b} \bar{z}^{l-c} \bar{w}^{g-l-d}$ certainly has this property, hence is in $\overline{\mathfrak{p}}$. 
Remark. In what follows we will use only the inhomogeneous part of the linear system whenever we talk about "this linear system".

We will use this linear system in the following way. If there exists a minimal isometric immersion of degree $g$ of an inhomogeneous lens space $L(p, q)$ into a sphere, its components can be described as in Lemma 4.3.6. The coefficients (the various $\left.a_{k i}, b_{k i}, c_{k i}, d_{k 1}\right)$ must satisfy a system of quadratic equations corresponding to the isometry condition. Hence if there is a solution to the quadratic system, there must also exist a solution to the above defined linear system. Our goal is to prove the contrapositive: if $g<\mathfrak{U}(p, q)$, then there exists no solution to the linear system, hence there is no solution to the quadratic system and a minimal isometric immersion of degree $g$ and image $L(p, q)$ cannot exist.

In general the solution of the linear system will not be unique. We will need the following:

Definition 4.3.8. Fix an inhomogeneous lens space $L(p, q)$ and a degree $g$. As explained above, associate with the isometry condition of a minimal immersion of $L(p, q)$ into a sphere an inhomogeneous linear system $A x=b$ where $A \in$ $M(6 g-4 \times n, \mathbb{R})$ and $b \in M(6 g-4 \times 1, \mathbb{R}), b \neq 0$. The number of equations derived by equating coefficients in equations (4.1.1) to (4.1.6) is $6 g-4$ and $n$ is the number of polynomials invariant under the appropriate group action. Define $m(g, p, q)$ to be the number of non-zero rows after row reducing $A$ and $n(g, p, q):=n$. Let

$$
\mathfrak{U}(p, q):=\inf _{g>0}\{g: m(g, p, q)-n(g, p, q) \leq 0\} .
$$

Note that our general assumption of $g<\mathfrak{U}(p, q)$ now assures that if the linear system has a solution it must be unique. This will be used in the following:

Proposition 4.3.9. Fix a degree $g$ and an inhomogeneous lens space $L(p, q)$. If $g<\mathfrak{U}(p, q)$ and if the system of quadratic equations has a solution $f$, then

$$
A \bar{A}_{i i}=B \bar{B}_{i i} \quad \text { for all } i, \quad \text { where } A \bar{A}_{i i}, B \bar{B}_{i i} \in \mathfrak{K} .
$$

Remark. Note that this proposition implies that if a component of $f$ contains a polynomial $\mathfrak{p}_{i}$ then $f$ must also contain $T\left(\mathfrak{p}_{i}\right)$. Also observe that we already know that if an immersion $f$ is a solution to the isometry equation, then so is $T(f)$. This follows from the fact that $T$ is an isometry of $S^{3}$.

Proof. First we will show that if we replace $f$ by $T(f)$, we obtain exactly the same linear system. Let $m$ be any monomial of degree $2(g-1)$. Denote by Coeff $[\mathfrak{p}, m]$ the coefficient of the monomial $m$ in the polynomial $\mathfrak{p}$. Using Lemma 4.3 .2 and equations 4.3.3 the coefficient comparison translates into

$$
\begin{aligned}
\operatorname{Coeff}\left[\sum_{k=1}^{n}\left(\frac{\partial f_{k}}{\partial z}\right)^{2}, T(m)\right] & =\operatorname{Coeff}\left[T\left[\sum_{k=1}^{n}\left(\frac{\partial f_{k}}{\partial z}\right)^{2}\right], m\right]=\operatorname{Coeff}\left[\sum_{k=1}^{n}\left[T\left(\frac{\partial f_{k}}{\partial z}\right)\right]^{2}, m\right] \\
& =\operatorname{Coeff}\left[\sum_{k=1}^{n}\left[\frac{\partial T\left(f_{k}\right)}{\partial \bar{z}}\right]^{2}, m\right] .
\end{aligned}
$$

Also we have that Coeff $\left[R_{1}, T(m)\right]=\operatorname{Coeff}\left[\bar{R}_{1}, m\right]$. The same holds true for the remaining equations. Therefore the system obtained by coefficient comparison for $f$ is the same as for $T(f)$, which proves the claim. The hypotheses of the proposition 
imply that a solution $f$ exists and that the corresponding linear system has a unique solution $L_{f}$. We obtain

$$
L_{f}= \begin{cases}A \bar{A}_{i i} & =r_{i} \\ B \bar{B}_{i i} & =s_{i} \\ C \bar{C}_{j j} & =t_{j} \\ D D_{11} & =u_{1} \text { for some } r_{i}, s_{i}, t_{j}, u_{1} \in \mathbb{R} \\ & \text { with } i=1, \cdots, n_{1}, j=1, \cdots, n_{2} \text { and } n_{1}+n_{2}+1=n .\end{cases}
$$

Applying $T$ to $f$ corresponds to interchanging the variables $A$ and $B$. We therefore obtain

$$
L_{T(f)}= \begin{cases}B \bar{B}_{i i} & =r_{i} \\ A \bar{A}_{i i} & =s_{i} \\ C \bar{C}_{j j} & =t_{j} \\ D D_{11} & =u_{1} \text { for some } r_{i}, s_{i}, t_{j}, u_{1} \in \mathbb{R} \\ & \text { with } i=1, \cdots, n_{1}, j=1, \cdots, n_{2} \text { and } n_{1}+n_{2}+1=n .\end{cases}
$$

But $L_{T(f)}=L_{f}$ as the two systems are the same and there is a unique solution. Therefore every solution under the hypothesis of the proposition has the following symmetric property:

$$
L_{f}= \begin{cases}A \bar{A}_{i i} & =B \bar{B}_{i i}=r_{i} \\ C \bar{C}_{j j} & =t_{j} \\ D D_{11} & =u_{1} \text { for some } r_{i}, t_{j}, u_{1} \in \mathbb{R} \\ & \text { with } i=1, \cdots, n_{1}, j=1, \cdots, n_{2} \text { and } n_{1}+n_{2}+1=n .\end{cases}
$$

Corollary 4.3.10. If the system of polynomial equations (4.1.1) to (4.1.6) has a solution $f$ of degree $g$ with $f$ invariant under that action of $G=\mathbb{Z}_{p}$ which generates the inhomogeneous lens space $L(p, q)$ and if $g<\mathfrak{U}(p, q)$, then the components of $f$ are linear combinations of $\mathfrak{p}_{i} \in P_{G}^{g} \cap T\left(P_{G}^{g}\right)$.

Proof. A set of polynomials is invariant under the action of $G$ if and only if the corresponding set of monomials is invariant under the action. Thus we can construct a basis for $P_{G}^{g}$ from the chosen basis of $P^{g}$ by taking only those polynomials $\left\{\mathfrak{p}_{i}\right\}$ which are invariant under $G$. Then Proposition 4.3 .9 implies that the components of $f$ contain only $\mathfrak{p}_{i} \in P_{G}^{g} \cap T\left(P_{G}^{g}\right)$.

\subsection{Finding $P_{G}^{g} \cap T\left(P_{G}^{g}\right)$.}

From section 4.2, we know that if we are looking for a minimal isometric immersion of $L(p, q)$, the monomials $m_{i}=z^{a_{i}} w^{b_{i}} \bar{z}^{c_{i}} \bar{w}^{d_{i}} \in M_{G}^{g}$ must satisfy $a_{i}+q b_{i}-c_{i}-$ $q d_{i} \equiv 0 \bmod p$. Hence in order to determine whether $T\left(m_{i}\right)=z^{c_{i}} w^{b_{i}} \bar{z}^{a_{i}} \bar{w}^{d_{i}} \in$ $M_{G}^{g}$, we need to solve $c_{i}+q b_{i}-a_{i}-q d_{i} \equiv 0 \bmod p$.

\section{Lemma 4.4.1.}

1. If $p$ is even, then

$$
\begin{aligned}
M_{G}^{g} \cap T\left(M_{G}^{g}\right)=\left\{m_{i}=z^{a_{i}} w^{b_{i}} \bar{z}^{c_{i}} \bar{w}^{d_{i}} \in M^{g}\right. \text { with } \\
\left.a_{i} \equiv c_{i} \quad \bmod \frac{p}{2} \quad \text { and } \quad b_{i} \equiv d_{i} \quad \bmod \frac{p}{2}\right\} .
\end{aligned}
$$


2. If $p$ is odd, then

$$
\begin{aligned}
M_{G}^{g} \cap T\left(M_{G}^{g}\right)= & \left\{m_{i}=z^{a_{i}} w^{b_{i}} \bar{z}^{c_{i}} \bar{w}^{d_{i}} \in M^{g}\right. \text { with } \\
& \left.a_{i} \equiv c_{i} \quad \bmod p \quad \text { and } \quad b_{i} \equiv d_{i} \quad \bmod p\right\} .
\end{aligned}
$$

Proof. Since the monomials $m_{i}=z^{a_{i}} w^{b_{i}} \bar{z}^{c_{i}} \bar{w}^{d_{i}} \in M_{G}^{g}$ have to satisfy both

$$
a_{i}+q b_{i}-c_{i}-q d_{i} \equiv 0 \quad \bmod p \quad \text { and } \quad c_{i}+q b_{i}-a_{i}-q d_{i} \equiv 0 \quad \bmod p,
$$

we obtain $2 q\left(b_{i}-d_{i}\right) \equiv 0 \bmod p$. As $p$ and $q$ are relatively prime this implies that $2\left(b_{i}-d_{i}\right) \equiv 0 \bmod p$. Hence

$$
\begin{array}{lll}
\left(b_{i}-d_{i}\right) \equiv 0 & \bmod \frac{p}{2} & \text { if } p \text { is even and } \\
\left(b_{i}-d_{i}\right) \equiv 0 & \bmod p & \text { if } p \text { is odd } .
\end{array}
$$

Observing that $a_{i}-c_{i} \equiv q\left(b_{i}-d_{i}\right) \bmod \frac{p}{2}$ in the case of $p$ even and $a_{i}-c_{i} \equiv$ $q\left(b_{i}-d_{i}\right) \bmod p$ for $p$ odd concludes the proof of Lemma 4.4.1.

Remark. The corresponding statement for polynomials is:

$$
\begin{aligned}
P_{G}^{g} \cap T\left(P_{G}^{g}\right)= & \{\text { polynomials constructed as in section } 4.3 \text { using monomials } \\
& \text { in } \left.M_{G}^{g} \cap T\left(M_{G}^{g}\right)\right\} .
\end{aligned}
$$

\subsection{No solutions to the isometry equations.}

Assuming $g<\mathfrak{U}(p, q)$ we saw in sections 4.2 to 4.4 that in order to construct a minimal isometric immersion of $L(p, q)$ into a sphere we need to use monomials $m_{i}=z^{a_{i}} w^{b_{i}} \bar{z}^{c_{i}} \bar{w}^{d_{i}}$ with $a_{i} \equiv c_{i} \bmod p$ and $b_{i} \equiv d_{i} \bmod p$ in the case of $p$ odd and with $a_{i} \equiv c_{i} \bmod \frac{p}{2}$ and $b_{i} \equiv d_{i} \bmod \frac{p}{2}$ for $p$ even.

Claim. If $g<\mathfrak{U}(p, q)$, there exists no solution to equation (4.1.3) and hence the system of isometry equations is insolvable.

Proof. Recall equation (4.1.3):

$$
\left(L_{3}:=\sum_{k=1}^{n} \frac{\partial f_{k}}{\partial z} \frac{\partial f_{k}}{\partial \bar{z}}\right)=\left(\frac{1}{2}(z \bar{z}+w \bar{w})^{g-1}+\frac{C}{4}(z \bar{z}+w \bar{w})^{g-2} z \bar{z}=: R_{3}\right) .
$$

We will show that the monomial $w^{g-1} \bar{w}^{g-1} \in R_{3}$ does not appear in the corresponding left hand side $L_{3}$. Since we solve a polynomial equation by equating coefficients, it will thus not be possible to solve equation (4.1.3). Let $f_{k}=\sum_{i=1}^{m} a_{i k} \mathfrak{p}_{i}$. Then

$$
\frac{\partial f_{k}}{\partial z}=\sum_{i=1}^{m} a_{i k} \frac{\partial \mathfrak{p}_{i}}{\partial z} \quad \text { and } \quad \frac{\partial f_{k}}{\partial \bar{z}}=\sum_{i=1}^{m} a_{i k} \frac{\partial \mathfrak{p}_{i}}{\partial \bar{z}}
$$

which leads to 


$$
\frac{\partial f_{k}}{\partial z} \frac{\partial f_{k}}{\partial \bar{z}}=\sum_{i=1}^{m} \sum_{j=i+1}^{m} a_{i k}^{2} \frac{\partial \mathfrak{p}_{i}}{\partial z} \frac{\partial \mathfrak{p}_{i}}{\partial \bar{z}}+a_{i k} a_{j k}\left(\frac{\partial \mathfrak{p}_{i}}{\partial z} \frac{\partial \mathfrak{p}_{j}}{\partial \bar{z}}+\frac{\partial \mathfrak{p}_{i}}{\partial \bar{z}} \frac{\partial \mathfrak{p}_{j}}{\partial z}\right)
$$

Again by substituting $\mathfrak{p}_{i}=\sum_{s=1}^{l} \lambda_{i s} m_{s}$, we obtain

$$
\begin{aligned}
L_{3}=\sum_{k=1}^{n} \frac{\partial f_{k}}{\partial z} \frac{\partial f_{k}}{\partial \bar{z}} & \\
=\sum_{k=1}^{n}\left\{\sum_{i=1}^{m} \sum_{j=i+1}^{m} \sum_{s=1}^{l} \sum_{r=s+1}^{l}\right. & \left(a_{i k}^{2} \lambda_{i s}^{2}+2 a_{i k} a_{j k} \lambda_{i s} \lambda_{j s}\right) \frac{\partial m_{s}}{\partial z} \frac{\partial m_{s}}{\partial \bar{z}} \\
& +\left(2 a_{i k}^{2} \lambda_{i s} \lambda_{i r}+2 a_{i k} a_{j k} \lambda_{i s} \lambda_{j r}\right) \frac{\partial m_{s}}{\partial z} \frac{\partial m_{r}}{\partial \bar{z}} \\
& \left.+\left(2 a_{i k}^{2} \lambda_{i s} \lambda_{i r}+2 a_{i k} a_{j k} \lambda_{i r} \lambda_{j s}\right) \frac{\partial m_{s}}{\partial \bar{z}} \frac{\partial m_{r}}{\partial z}\right\}
\end{aligned}
$$

All monomials on the left hand side, $L_{3}$, of the equation are of the form

$$
\frac{\partial m_{s}}{\partial z} \cdot \frac{\partial m_{r}}{\partial \bar{z}} \quad \text { for any } s \text { and } r .
$$

Assume $g$ odd. Since all of these monomials must be invariant under $T$ by Lemma 4.4.1, we can uniquely express $m_{s}$ and $m_{r}$ in the following way:

$$
\begin{aligned}
& m_{s}=z^{\mu_{s} p+c_{s}} w^{\nu_{s} p+d_{s}} \bar{z}^{c_{s}} \bar{w}^{d_{s}} \\
& m_{r}=z^{\mu_{r} p+c_{r}} w^{\nu_{r} p+d_{r}} \bar{z}^{c_{r}} \bar{w}^{d_{r}},
\end{aligned}
$$

where $\mu_{s}, \nu_{s}, \mu_{r}$ and $\nu_{r}$ are integers and $0 \leq \mu_{s} p+c_{s}, 0 \leq \nu_{s} p+d_{s}, 0 \leq c_{s}, 0 \leq$ $d_{s}, 0 \leq \mu_{r} p+c_{r}, 0 \leq \nu_{r} p+d_{r}, 0 \leq c_{r}, 0 \leq d_{r}$. In order to obtain nonzero partial derivatives we must have that

$$
\mu_{s} p+c_{s}>0 \quad \text { and } \quad c_{r}>0 .
$$

Thus

$$
\begin{aligned}
& \frac{\partial m_{s}}{\partial z}=\left(\mu_{s} p+c_{s}\right) z^{\mu_{s} p+c_{s}-1} w^{\nu_{s} p+d_{s}} \bar{z}^{c_{s}} \bar{w}^{d_{s}} \\
& \frac{\partial m_{r}}{\partial \bar{z}}=c_{r} z^{\mu_{r} p+c_{r}} w^{\nu_{r} p+d_{r}} \bar{z}^{c_{r}-1} \bar{w}^{d_{r}}
\end{aligned}
$$

and

$$
\frac{\partial m_{s}}{\partial z} \cdot \frac{\partial m_{r}}{\partial \bar{z}}=c_{r}\left(\mu_{s} p+c_{s}\right) z^{\left(\mu_{s}+\mu_{r}\right) p+c_{s}+c_{r}-1} w^{\left(\nu_{s}+\nu_{r}\right) p+d_{s}+d_{r}} \bar{z}^{c_{s}+c_{r}-1} \bar{w}^{d_{s}+d_{r}} .
$$

Since we are looking for monomials $m_{s}$ and $m_{r}$ such that

we need that

$$
\frac{\partial m_{s}}{\partial z} \cdot \frac{\partial m_{r}}{\partial \bar{z}}=w^{g-1} \cdot \bar{w}^{g-1}
$$

$$
\begin{aligned}
\left(\mu_{s}+\mu_{r}\right) p+c_{s}+c_{r}-1 & =0, \\
c_{s}+c_{r}-1 & =0, \\
\left(\nu_{s}+\nu_{r}\right) p+d_{s}+d_{r} & =g-1, \\
d_{s}+d_{r} & =g-1 .
\end{aligned}
$$

These equations directly imply that $\left(\mu_{s}+\mu_{r}\right) p=0$ and $\left(\nu_{s}+\nu_{r}\right) p=0$. Since $p \neq 0$, we obtain 


$$
\begin{aligned}
\mu_{s} & =-\mu_{r}, \\
\nu_{s} & =-\nu_{r}, \\
c_{s}+c_{r} & =1, \\
d_{s}+d_{r} & =g-1 .
\end{aligned}
$$

Since $c_{s}$ and $c_{r}$ are non-negative integers, the only solutions of $c_{s}+c_{r}=1$ are

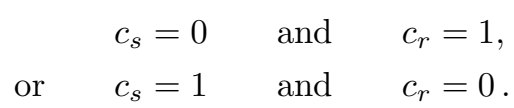

But the case (4.5.2) is not possible since we assumed that $c_{r}>0$. The case (4.5.1) is also not possible, since with condition (4.5.0) it implies that $\mu_{s}>0$. But, then $\mu_{r} p+c_{r}=-\mu_{s} p+1<1$. As $\mu_{r} p+c_{r} \geq 0$ this implies that $-\mu_{s} p+1=0$ which is not possible, as $p>1$.

For case (2) of Lemma 4.4.1, i.e. $g$ even, repeat the same argument replacing $p$ by $\frac{p}{2}$.

Conclusion. There do not exist monomials $m_{s}, m_{r} \in M_{G}^{g} \cap T\left(M_{G}^{g}\right)$ such that

$$
\frac{\partial m_{s}}{\partial z} \cdot \frac{\partial m_{r}}{\partial \bar{z}}=w^{g-1} \cdot \bar{w}^{g-1}
$$

Thus equation (4.1.3) is not solvable. Therefore it is not possible to solve the isometry equation for a minimal immersion of degree $g$ of $L(p, q)$ into a sphere if $g<\mathfrak{U}(p, q)$.

This concludes the proof of Theorem 2 .

\section{REFERENCES}

[C] E. Calabi, Minimal immersions of surfaces in euclidean spheres, J. Diff. Geom. 1967 (1), 111-125. MR 38:1616

[DW1] M. doCarmo and N.Wallach, Representations of compact groups and minimal immersions into spheres, J. Diff. Geom. 1970, no. 4, 91 - 104. MR 42:1013

[DW2] Minimal immersions of spheres into spheres, Annals of Math. 1971, no. 93, 43 - 62. MR 43:4048

[DZ] D. DeTurck and W.Ziller, Minimal isometric immersions of spherical space forms into spheres, Comm. Math. Helv. 1992, no. 67, 428-458. MR 93f:53050

[E] N.Ejiri, Totally real submanifolds in a 6-sphere, Proc. Amer. Math. Soc. 1981, no. 83, 759-763. MR 83a:53033

[L] P. Li, Minimal immersions of compact irreducible homogeneous Riemannian manifolds, J. Diff. Geom. 1984, no. 16, 337- 358. MR 83a:53057

[Ma1] K. Mashimo, Minimal immersions of 3-dimensional spheres into spheres, Osaka J. Math. 1984, no. 2, 721 - 732. MR 86j:53040

[Ma2] Homogeneous totally real submanifolds in $S^{6}$, Tsukuba J. Math. 1985, no. 9, 185 - 202. MR 86j:53083

[Mr] J.D. Moore, Isometric immersions of space forms into space forms, Pacific J. Math. 1976, no. 40, 157 - 166. MR 46:4442

[M] Y. Muto, The space $W_{2}$ of isometric minimal immersions of the three-dimensional sphere into spheres, Tokyo J. Math. 1981, no. 7, 105- 115. MR 86e:53042

[S] P. Scott, The geometries of 3-manifolds, Bull. London. Math. Soc. 1983, no. 5, 401487. MR 84m:57009

[T] T. Takahashi, Minimal immersions of Riemannian manifolds, J. Math. Soc. Japan 1966, no. 18, 380- 385. MR 33:6551

[To] G. Toth, Eigenmaps and the space of minimal immersions between spheres, Indiana Univ. Math. J. 1994, no. 4. 
[TS] W. Threlfall und H. Seifert, Topologische Untersuchung der Diskontinuitätsbereiche endlicher Bewegungsgruppen des dreidimensionalen sphärischen Raumes, Math. Annalen 1930, no. 104, 1-70.

[V] N. J. Vilenkin, Special Functions and the Theory of Group Representations, American Mathematical Society, 1968. MR 37:5429

[W] J. Wolf, Spaces of Constant Curvature, McGraw-Hill, 1967. MR 36:829

[Wa] N. Wallach, Extension of locally defined minimal immersions into spheres, Arch. Math. 1970, no. 21, 210-213. MR 42:6759

[WZ] M. Wang and W. Ziller, On isotropy irreducible Riemannian manifolds, Acta Math. 1991, no. 166, 223-261. MR 92b:53078

Department of Mathematics, Oregon State University, Corvallis, Oregon 97331

E-mail address: tine@math.orst.edu 